\title{
Atteinte urinaire et pseudo-obstruction intestinale révélatrice d'un lupus érythémateux systémique à propos d'une observation
}

\author{
Urinary disease and intestinal pesudo-obstruction revealing a systemic lupus erythematous \\ about an observation \\ DIABY LM ${ }^{1}$, KANE AST ${ }^{2}$; SANOGO A ${ }^{1}$, MAIGA AS ${ }^{2}$; CAMARA Y ${ }^{1}$; BAGAYOKO DK ${ }^{1}$. \\ ${ }^{1}$ Service Médecine; Infirmerie Hôpital Militaire de Kati. \\ ${ }^{2}$ Service Odontologie; Infirmerie Hôpital Militaire de Bamako
}

Correspondant: Dr Diaby Ladji Mohamed, Service de médecine; Infirmerie Hôpital Militaire de Kati Email: ladjimohameddiaby@gmail.com

\section{RESUME}

Introduction : Le lupus érythémateux systémique est une connectivite fréquente chez les femmes en activité génitale. Au cours du lupus érythémateux systémique, la pseudo-obstruction intestinale est l'une des graves complications rares. Ainsi l'objectif de cet article est de présenter un cas clinique d'atteinte urinaire et pseudo-obstruction intestinale révélatrice d'un lupus érythémateux systémique. Observation : Une patiente âgée de 28 ans mariée, de Nationalité Guinéenne admise pour une diarrhée fébrile, asthénie, météorisme, associée à des douleurs articulaire et abdominale nécessitant une consultation et des bilans sans succès.Une échographie abdominale qui objective un épaississement diffus de la paroi colique et des anses grêles ainsi qu'une ascite de moyenne abondance. La coloscopie révèle un aspect inflammatoire spécifique de la muqueuse iléale.

Conclusion : La pseudo-obstruction intestinale peut être inaugurale ou concomitante du lupus érythémateux systémique.

Mots clés : Atteinte urinaire, pseudo-obstruction intestinale, lupus érythémateux

\section{ABSTRACT}

Introduction: Systemic lupus erythematosus is a common connective tissue disease in women with genital activity. During lupus disease intestinal pseudo-obstruction is one of the rare serious complications. Thus the objective of this article is to present a clinical case of urinary involvement and intestinal pseudo-obstruction revealing a systemic lupus erythematosus.Observation: A 28-year-old female patient of Guinean nationality admitted for febrile diarrhea, asthenia, meteorism, associated with joint and abdominal pain requiring consultation and balance sheets without success. An abdominal ultrasound showing diffuse thickening of the colonic wall and small loops as well as ascites of average abundance. Colonoscopy reveals a specific inflammatory aspect of the ileal mucosa.

Conclusion: The intestinal pseudo-obstruction may be inaugural or concomitant with systemic lupus erythematosus.

Key words: Urinary involvement, intestinal pseudoobstruction, lupus erythematosus.

\section{INTRODUCTION}

Le lupus érythémateux systémique (LES) est une affection auto-immune complexe dont les manifestations cliniques sont nombreuses et l'évolution très hétérogène.L'origine de cette affection chronique multi-systémique est inconnue bien que des facteurs génétiques, environnementaux, hormonaux et infectieux jouent un rôle dans la pathogénie de l'affection. Le lupus érythémateux systémique est une connectivite fréquente chez les femmes en activité génitale. $\mathrm{Au}$ cours du lupus érythémateux systémique la pseudo-obstruction intestinale est l'une des graves complications rares [1] .Les tests immunologiques, aussi sophistiqués soient-ils, manquent souvent d'une complète spécificité et peuvent rester définitivement négatifs malgré un tableau clinique caricatural. Ainsi les anticorps anti-ADN natif, s'ils restent le meilleur critère biologique du lupus érythémateux systémique, ne suffisent pas à eux seuls pour porter le diagnostic de lupus érythémateux systémique (LES). Ils ont été signalés au cours d'autres affections [1] et ne sont probablement pas la cause exclusive des mécanismes pathogéniques. Leur présence est loin d'être constante. La moins mauvaise définition serait clinico-biologique. C'est l'attitude de Dubois qui définit le lupus érythémateux systémique comme un syndrome clinique de cause inconnue caractérisé par une atteinte systémique et par une évolution par poussées, atteignant un ou plusieurs appareils, entrecoupée de rémissions multiples. Le diagnostic pourra être confirmé chez la majorité des malades par la découverte d'autoanticorps dirigés contre les 
constituants du noyau des cellules (acides nucléiques et protéines).Ainsi l'objectif de cet article est de présenter un cas clinique d'atteinte urinaire et pseudo-obstruction intestinale révélatrice d'un lupus érythémateux systémique. [1]

\section{OBSERVATION}

Il s'agit d'une patiente âgée de 28 ans mariée ; de Nationalité Guinéenne admise le 22 février 2014 pour une diarrhée fébrile, asthénie, météorisme, associée à des douleurs articulaire et abdominale nécessitant une consultation et des bilans sans succès. L'examen clinique était pauvre en dehors d'une déshydratation et d'une défense à la palpation de l'abdomen. Une échographie abdominale qui objective un épaississement diffus de la paroi colique et des anses grêles ainsi qu'une ascite de moyenne abondance. La coloscopie révèle un aspect inflammatoire spécifique de la muqueuse iléale. Le transit du grêle est très ralenti. La patiente abandonne le traitement médical au profit d'un traitement traditionnel. Elle est revenue en janvier 2015 pour une polyarthrite, bilatérale, périphérique et symétrique (voir figure 1) avec alopécie diffuse, surtout des odèmes des membres inférieurs et du visage (voir figure 2) révélant un syndrome néphrotique (albumine 20,1 g/L, protéinurie $4,37 \mathrm{~g} / \mathrm{J})$. La créatinine est à $70 \mu \mathrm{mol} / \mathrm{L}$. anti-ADN 67UI/ml (Normal <15). Le complément est effondré. La ponction biopsie rénale et histologie confirme le diagnostic de néphropathie lupique stade II $+\mathrm{V}$ de l'OMS. Une corticothérapie orale est instaurée à la dose $1 \mathrm{mg} / \mathrm{kg} / \mathrm{j}$. En aout 2015 la patiente interrompt son traitement et quarante-cinq jours plus tard, elle présente une récidive des douleurs articulaires diffuses, de la diarrhée et des douleurs abdominales ainsi qu'une dysurie. On constate un tableau d'insuffisance rénale aiguë (créatinine $112 \mu \mathrm{mol} / \mathrm{L}$ ). L'échographie révèle une hydronéphrose bilatérale, avec l'épaississement de la paroi vésicale et des anses grêles. La cystoscopie révèle une capacité vésicale réduite et d'épaisses trabéculations, avec une histologie aspécifique. La réalisation d'une corticothérapie s'impose avec 3 bolus de solumédrol et la pose d'une sonde vésicale permettent la résolution de la symptomatologie digestive et de l'insuffisance rénale en 21 jours. Un traitement à base d'hydroxychloroquine de $1 \mathrm{~g} / \mathrm{j}$ est instauré, associé à l'azathioprine $100 \mathrm{mg} / \mathrm{J}$. A trois mois d'azathioprine, sous cortancyl (prednisolone) 20 $\mathrm{mg} / \mathrm{j}$, et $1 \mathrm{~g}$ d'hydroxychloroquine la patiente est toujours asymptomatique sur le plan digestif mais l'hydronéphrose est restée inchangée. La fonction rénale était normale. La symptomatologie digestive a disparu avec la persistance de l'uretro- hydronephrose.

\section{DISCUSSION}

Reins : Les atteintes rénales constituent un pronostique majeur important. Leur fréquence comprise entre 35 et $55 \%$ (plus élevée si l'on se fonde sur les données de biopsies rénales systématiques). L'atteinte rénale survient en règle dans les premières années d'évolution. Une surveillance régulière s'impose. La ponction-biopsie rénale $(\mathrm{PBR})$ est indiquée en cas de protéinurie supérieure à $0,5 \mathrm{~g} / 24 \mathrm{~h}$. Les lésions sont principalement glomérulaires, mais aussi tubulointerstitielles et parfois vasculaires ; elles coexistent fréquemment sur une même biopsie [2]. On distingue : les lésions actives susceptibles de régresser sous traitement ; les lésions inactives irréversibles ; chacune avec un indice quantitatif. L'Organisation Mondiale de la Santé (OMS) reconnaît six classes : Glomérule normal (classe I), cet aspect est rare ; Glomérulonéphrite mésangiale pure (classe II) de pronostic favorable ; -Glomérulonéphrite segmentaire et focale (classe III) : avec des lésions nécrotiques et prolifératives partielles de capillaires (moins de $50 \%$ des glomérules), une protéinurie modérée, une évolution ultérieure vers une forme diffuse non exceptionnelle ; - Glomérulonéphrite proliférative diffuse (classe IV) : c'est la forme la plus fréquente et la plus grave avec une protéinurie franche, souvent un syndrome néphrotique impur associant hématurie microscopique, HTA et insuffisance rénale. Les glomérules sont touchés à des degrés divers : nécrose, prolifération des cellules mésangiales et endothéliales, dépôts endomembraneux, prolifération épithéliale (croissants extra capillaires, signe de gravité), dépôts granuleux d'IgG, IgM, IgA, ou de complément ; Glomérulonéphrite extra membraneuse (classe V) : c'est un syndrome néphrotique avec hématurie microscopique, sans HTA ni insuffisance rénale. Les parois des capillaires glomérulaires sont épaissies de façon diffuse et régulière par des dépôts immuns ; sclérose glomérulaire (classe VI), d'autonomie discutée.

Vessie : Elle peut être le siège d'une inflammation spécifiquement lupique [2], Après les premiers cas rapportés par De la Sema et coll., en 1981 [2], d'autres observations ont été publiées [5]. Les signes cliniques sont dominés par une cystite parfois inaugurale mais jamais isolée, accompagnée très fréquemment de signes digestifs (diarrhée, malabsorption, douleurs, iléus). Les explorations radiologiques mettent en évidence une petite vessie aux parois épaissies, à la capacité réduite. Il peut 
s'agir d'un mode de rechute de la maladie. Un retentissement en amont est possible avec uretérohydronéphrose. Les corticostéroïdes à fortes doses sont efficaces permettant une guérison clinique et anatomique de la cystite [6,7]. Le rôle des immunosuppresseurs, notamment le cyclophosphamide, semble pouvoir être exclu et Boye [9] a incriminé le rôle de complexes immuns déposés dans la paroi vésicale. Il s'agit toujours de lupus graves, 3 des 5 malades décrits étant décédés. Enfin, on a signalé 1 cas d'insuffisance rénale par sténose urétérale lombo-iliaque au cours d'un lupus avec syndrome des anti phospholipides.

Manifestations digestives: Cliniquement, les manifestations gastro-intestinales sont fréquentes. Elles recouvrent des entités très variables de pronostic souvent très différent. Tout le problème est de ne pas laisser passer une indication chirurgicale $[7,8]$. L'anorexie, les nausées et les vomissements (11 à 53 p. 100) accompagnent fréquemment une poussée de la maladie. Il peut s'y associer une diarrhée (4 à 12 p. 100) dont l'origine infectieuse est parfois démontrée [9]. Pour les Hémorragies digestives, elles sont rapportées dans 1,5 à 6,3 p. 100 des cas; leur origine est parfois médicamenteuse (corticoïdes, aspirine) mais aussi lupique par ulcérations ischémiques coliques ou intestinales, exceptionnellement par infestation helminthique massive (anguillulose : 1 cas personnel). Elles posent un problème difficile et fréquent (6 à 34 p. 100) chez les lupiques. Multiples sont les causes possibles regroupées dans le tableau I. L'apparition d'une ascite [9] peut relever d'un mécanisme identique à celui des péricardites et pleurésies, avec cellules LE dans le liquide. Son apparition est parfois insidieuse, sans douleur [9]. Elle peut être révélatrice [9]. En fait, il faudra éliminer une péritonite par perforation $[\mathbf{6}$, $\mathbf{7 , 8 , 9 ]}$, une ulcération, une infection à pyogènes ou tuberculeuse, voire une insuffisance cardiaque, rénale ou hépatique ou un hémopéritoine par rupture d'organe plein. C'est dire que la ponction exploratrice sera systématique avec-ensemencement sur tous milieux. On aura parfois la surprise de trouver un hémopéritoine [9], Des observations isolées de complications plus rares ont été rapportées : entéropathie exsudative [9] régressant sous corticothérapie ; syndrome de malabsorption avec atrophie villositaire intestinale; pneumatose kystique [9], parfois compliquée de pneumopéritoine [9], péri hépatite [9]. Toutes ces manifestations ne doivent pas faire oublier le pronostic souvent redoutable de certaines complications : pancréatite [9], infarctus mésentérique [9], hémopéritoine [9] et perforations intestinales qui relèvent d'un même mécanisme de vascularite mésentérique. Ainsi, Zizic [6] rapporte 5 cas de perforations coliques parfois multiples dont 4 mortelles, soit 27 p. 100 d'un total de 15 décès qu'il a eu à déplorer dans sa série (voir tableau I).

\section{CONCLUSION}

Le lupus est une maladie auto-immune, fréquente chez la femme en activité génitale. Elle est d'origine multifactorielle pouvant entrainé une altération de la qualité de vie voire engagé le pronostic vital. La pseudo-obstruction intestinale peut être inaugurale ou concomitante du lupus érythémateux systémique. Le diagnostic précoce a pour objectif d'instaurer rapidement le traitement immunosuppresseur adapté ; d'éviter les complications systémiques et surtout de prévenir un geste chirurgical inapproprié.

\section{Conflit d'intérêt : aucun}

\section{Références}

1-EPSTEIN WV, TAN M, EASTERBROOK M.

Serum antibody to ARN and ADN in patients with uveitis. N Engl J Med, 1971, 285: 1502-1506.

2-DE LA SERNA AR, ALARCON-SEGOVIA D. Chronic interstitial cystitis as an initial major manifestation of systemic lupus erythematosus. J Rheumatol, 1981, 8:808-810.

3-ORTH RW, WEIJMAN MH, COHEN AH ct coll. Lupus cystitis: primary bladder manifestations of systemic lupus erythematosus. Ann Intern Med, 1983, 98: 323-326.

4-GOUPILLE PH, JEANNOU J, VALAT JP. Lupus cystitis improved with oral prednisolone therapy. J Rheumatol, 1996, 23: 1667.

5-SEGAWA C, WADA T, YOKOYAMA $\mathrm{H}$. Efficacy of steroid pulse therapy in lupus cystitis. J Rheumatol, 1995, 22: 2373-2374.

6-ZIZIC TM, CLASSEN JN, STEVENS MB. Acute abdominal complications of systemic lupus erythematosus and polyarteritis nodosa. Am J Med, 1982, 73: 525-531.

7-ZIZIC TM. Gastrointestinal manifestations. In: $\mathrm{PH}$ Schur. The clinical management of systemic lupus erythematosus. New York, Grune et Stratton, 1983: 153-166.

8-SÇHOUSDOE JT, KOCH AE, CHANG RW. Chronic lupus peritonitis with ascites: review of the literature with a case report. Semin Arthritis Rheum, 1988, 18: 121-126. ( c' est un article)

9-KAHN. M.F, MEYER. O, PELTIER. A. P, PIETTE J.C. Maladies et syndromes systémiques $4^{\text {ieme }}$ edition, paris, 2001, $2^{\text {ieme }}$ tirage 2001, 131-168. 
Tableau I. les causes de douleurs abdominales.

\begin{tabular}{|c|c|}
\hline Causes chirurgicales & Causes médicales \\
\hline $\begin{array}{lll}\text { Infarctus intestinal (vascularite } & \text { ou } \\
\text { anticoagulant circulant) } & & \\
\end{array}$ & Ascite \\
\hline $\begin{array}{lll}\begin{array}{l}\text { Perforation } \\
\text { gastroduodénal }\end{array} & \text { iatrogène d'ulcère } \\
\end{array}$ & Péritonite aseptique \\
\hline Perforation colique ou jéjunale par artérite & Hémopéritoine \\
\hline Invagination intestinale & Entérite régionale \\
\hline Pneumatose cystoïde intestinale & Entéropathie exsudative \\
\hline Hémobilie & Hématome perirenal \\
\hline Rupture spontanée hépatique, splénique & $\begin{array}{l}\text { Pseudo-obstruction intestinale chronique } \\
\text { (POIC) }\end{array}$ \\
\hline Pseudo-anévrysme pancréatique & Infection intestinale, urinaire \\
\hline Pancréatite & Fibrose rétro péritonéale \\
\hline $\begin{array}{l}\text { Vascularite } \\
\text { - thrombose artérielle (anticoagulant } \\
\text { circulant) } \\
\text { - cortico-induite? } \\
\text { - induite par l'azathioprine? }\end{array}$ & $\begin{array}{l}\text { Douleurs projetées } \\
\text { - pleurésie } \\
\text { - péricardite } \\
\text { - angor }\end{array}$ \\
\hline
\end{tabular}

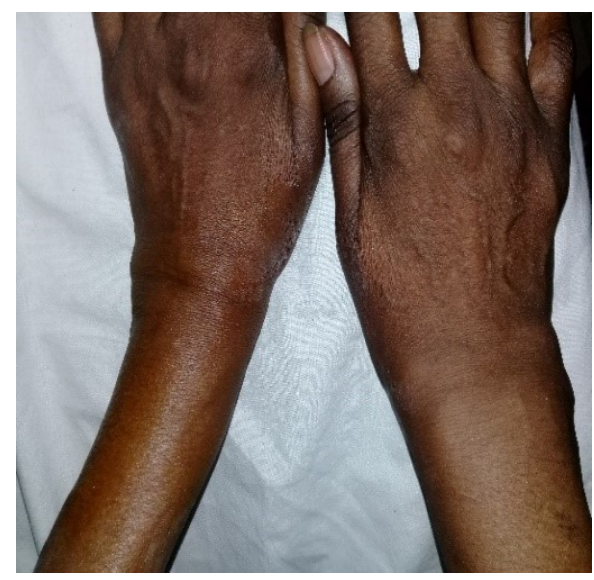

Figure 1. Arthrite des poignets avec synovite

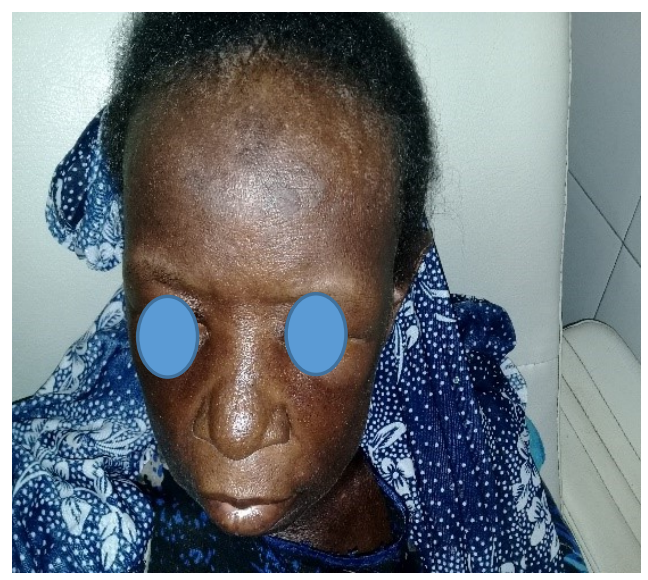

Figure 2. visage avec des lésions dermatologiques (taches hypochromes et lésions en lunette) et alopécie 